\title{
A!
}

This is an electronic reprint of the original article.

This reprint may differ from the original in pagination and typographic detail.

Liu, Jiandang; Jin, L.; Jiang, Z.; Liu, Laijun; Himanen, L.; Wei, J.; Zhang, Nan; Wang, Dawei; Jia, C. L.

Understanding doped perovskite ferroelectrics with defective dipole model

Published in:

Journal of Chemical Physics

DOI:

$10.1063 / 1.5051703$

Published: 28/12/2018

Document Version

Publisher's PDF, also known as Version of record

Please cite the original version:

Liu, J., Jin, L., Jiang, Z., Liu, L., Himanen, L., Wei, J., Zhang, N., Wang, D., \& Jia, C. L. (2018). Understanding doped perovskite ferroelectrics with defective dipole model. Journal of Chemical Physics, 149(24), [244122]. https://doi.org/10.1063/1.5051703

This material is protected by copyright and other intellectual property rights, and duplication or sale of all or part of any of the repository collections is not permitted, except that material may be duplicated by you for your research use or educational purposes in electronic or print form. You must obtain permission for any other use. Electronic or print copies may not be offered, whether for sale or otherwise to anyone who is not an authorised user. 


\section{Understanding doped perovskite ferroelectrics with defective dipole model}

Cite as: J. Chem. Phys. 149, 244122 (2018); https://doi.org/10.1063/1.5051703

Submitted: 11 August 2018 . Accepted: 05 December 2018 . Published Online: 31 December 2018

J. Liu, L. Jin (D), Z. Jiang, L. Liu (D), L. Himanen (D), J. Wei, N. Zhang (D), D. Wang, and C.-L. Jia

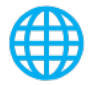

\section{ARTICLES YOU MAY BE INTERESTED IN}

Perspective: Nonlinear approaches to structure and dynamics of soft materials

The Journal of Chemical Physics 149, 240901 (2018); https://doi.org/10.1063/1.5065412

Relating free energy and open-circuit voltage to disorder in organic photovoltaic systems The Journal of Chemical Physics 149, 244123 (2018); https://doi.org/10.1063/1.5050506

Symmetry changes during relaxation process and pulse discharge performance of the $\mathrm{BaTiO}_{3}-\mathrm{Bi}\left(\mathrm{Mg}_{1 / 2} \mathrm{Ti}_{1 / 2}\right) \mathrm{O}_{3}$ ceramic

Journal of Applied Physics 124, 054101 (2018); https://doi.org/10.1063/1.5030381

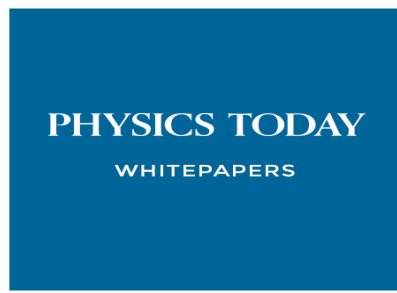

ADVANCED LIGHT CURE ADHESIVES

Take a closer look at what these environmentally friendly adhesive systems can do
READ NOW

PRESENTED BY 8. MASTERBOND" 


\title{
Understanding doped perovskite ferroelectrics with defective dipole model
}

\author{
J. Liu, ${ }^{1}$ L. Jin, ${ }^{2}$ Z. Jiang, ${ }^{3, a)}$ L. Liu, ${ }^{4}$ L. Himanen, ${ }^{5}$ J. Wei, ${ }^{2}$ N. Zhang, ${ }^{2}$ D. Wang,,${ }^{3, b)}$ \\ and C.-L. Jia ${ }^{3,6}$ \\ ${ }^{1}$ State Key Laboratory for Mechanical Behavior of Materials, School of Materials Science \\ and Engineering, Xi'an Jiaotong University, Xi'an 710049, China \\ ${ }^{2}$ Electronic Materials Research Laboratory, Key Laboratory of the Ministry of Education \\ and International Center for Dielectric Research, School of Electronic and Information Engineering, \\ $X i$ 'an Jiaotong University, Xi'an 710049, China \\ ${ }^{3}$ School of Microelectronics and State Key Laboratory for Mechanical Behavior of Materials, \\ Xi'an Jiaotong University, Xi'an 710049, China \\ ${ }^{4}$ College of Materials Science and Engineering, Guilin Univeristy of Technology, Guilin 541004, China \\ ${ }^{5}$ Department of Applied Physics, Aalto University, Espoo 00076, Finland \\ ${ }^{6}$ Peter Grünberg Institute and Ernst Ruska Center for Microscopy and Spectroscopy with Electrons, \\ Research Center Jülich, D-52425 Jülich, Germany
}

(Received 11 August 2018; accepted 5 December 2018; published online 31 December 2018)

\begin{abstract}
While doping is widely used for tuning physical properties of perovskites in experiments, it remains a challenge to exactly know how doping achieves the desired effects. Here, we propose an empirical and computationally tractable model to understand the effects of doping with $\mathrm{Fe}$-doped $\mathrm{BaTiO}_{3}$ as an example. This model assumes that the lattice sites occupied by a Fe ion and its nearest six neighbors lose their ability to polarize, giving rise to a small cluster of defective dipoles. Employing this model in Monte Carlo simulations, many important features such as reduced polarization and the convergence of phase transition temperatures, which have been observed experimentally in acceptor doped systems, are successfully obtained. Based on microscopic information of dipole configurations, we provide insights into the driving forces behind doping effects and propose that active dipoles, which exist in proximity to the defective dipoles, can account for experimentally observed phenomena. Close attention to these dipoles is necessary to understand and predict doping effects. Published by AIP Publishing. https://doi.org/10.1063/1.5051703
\end{abstract}

\section{INTRODUCTION}

For perovskite ferroelectrics, doping chemical elements is an important way to improve or modify their properties and performances. ${ }^{1-4}$ In many cases, it appears that minuscule doping already has strong effects on the resulting materials. While there is a large amount of literature on exploiting doping effects experimentally, the nature and cause of the observed effects are not fully understood, and several possible factors are proposed to explain experimental results. The effects of doping induced oxygen vacancies and ferroelectric domains, among others, are often believed to play important roles. ${ }^{5-14}$ In fact, the exact mechanism of doping effects is hard to identify by working backward (i.e., to deduce directly from experimental results); thus, a lot of theoretical modeling is needed in this process. Usually, the most reliable method to calculating doping effects is the first principle calculation. However, this method has practical difficulty for minuscule doping where too many atoms are needed in the simulations, which causes heavy computational burden. Here we focus on the iron-doped $\mathrm{BaTiO}_{3}$ and consider this problem in an opposite direction. ${ }^{15}$

\footnotetext{
a)Present address: School of Physics and Optoelectronic Engineering, Ludong University, Yantai 264025, China.

b)Author to whom correspondence should be addressed: dawei.wang@ xjtu.edu.cn
}

In other words, we first propose a computationally tractable model regarding how doping works, and then with the effective Hamiltonian approach, ${ }^{16-19}$ we explore the consequences of the proposed model. Comparing the simulated results for samples with and without doping to experimental results, one will gain better understanding of the nature of doping effects.

Barium titanate $\left(\mathrm{BaTiO}_{3}\right)$, a typical ferroelectric oxide with perovskite $\mathrm{ABO}_{3}$ structure (with $\mathrm{Ba}$ on the $\mathrm{A}$ site and Ti on the $\mathrm{B}$ site), has been widely investigated due to its high dielectric permittivity, excellent electrical properties, and environmental friendliness. ${ }^{20,21}$ Doping of $\mathrm{BaTiO}_{3}$-based materials with different chemical elements has been an attractive topic of research, ${ }^{22-27}$ with the goal to improve material performance. One important line of research is to dope rare earth elements into $\mathrm{BaTiO}_{3}$. For instance, Yasmin et al. found that the Ce-doped $\mathrm{BaTiO}_{3}$ has a dielectric permittivity as high as 2050 and a decreased Curie temperature, $T_{C}=313 \mathrm{~K}^{27}$ Ganguly et al. reported that at $10 \mathrm{kHz}$, the dielectric permittivity for $\left(\mathrm{Ba}_{1-x} \mathrm{La}_{2 x / 3}\right) \mathrm{TiO}_{3}(x=0.1)$ can reach 10400 at $T_{C} \simeq 168 \mathrm{~K}^{28} \mathrm{Ba}\left(\mathrm{Zr}_{x} \mathrm{Ti}_{1-x}\right) \mathrm{O}_{3}$ ceramics, where the $\mathrm{B}$-site $\mathrm{Ti}$ is substituted with $\mathrm{Zr}$, shows enhanced remnant polarization and field piezoelectric strain coefficient $d_{33},{ }^{22}$ as well as interesting dielectric properties on the subterahertz frequency range. ${ }^{19} \mathrm{La}$ and $\mathrm{Zr}$ co-doped $\mathrm{BaTiO}_{3}$ can obtain a dielectric permittivity as high as $36000 .{ }^{23}$ In addition, doping 
transitional metal ions could also achieve novel ferromagnetic properties with a saturation magnetization value as large as $0.012 \mathrm{emu} / \mathrm{g} .^{24,29}$

In experimental investigations of doped systems, three phenomena are often observed as the consequences of doping: (i) strong change of the hysteresis loop, (ii) diffused and/or smeared dielectric permittivity with respect to the temperature, and (iii) variations of phase transition temperatures. ${ }^{24,25,30-37}$ Moreover, it is also known from experiments that doping $\mathrm{Fe}$ or $\mathrm{Mn}$ into $\mathrm{BaTiO}_{3}$ in general makes ferroelectric materials easier to reverse. ${ }^{38-41}$ Since the doping effects can be significant even with minuscule doping, the origin of such effects naturally attracts great scientific attention. Different explanations have been proposed to understand the mechanism of doping effects, including (i) oxygen vacancies and free charges on the doped lattice point, ${ }^{10,12-14,41-43}$ (ii) local strains, ${ }^{44-46}$ and (iii) domains induced by dopants. ${ }^{9-11,47}$ In the present work, we propose a computationally tractable model where the dipoles associated with Fe-doped lattice sites and their nearest neighbors are suppressed. We apply this model to mimic Fe-doped $\mathrm{BaTiO}_{3}$ and perform first-principles-based Monte Carlo (MC) simulations, showing that the simulated results can account for many experimentally observed phenomena. Through the aforementioned approach, we hope to better understand the most important factors that determine the properties of $\mathrm{BaTiO}_{3}$ doped with iron.

This paper is organized as follows: In Sec. II, we introduce the defective dipole model and the effective Hamiltonian method for numerical simulation. In Secs. III and IV, we apply this model to samples mimicking Fe-doped $\mathrm{BaTiO}_{3}$ and numerically obtain the results of doping. In Sec. V, we propose the concept of active dipoles and use it (along with dipole distributions) to explain doping effects. Finally, in Sec. VI, we present a brief conclusion.

\section{METHOD}

The transition metal ions we are concerned with, including $\mathrm{Fe}, \mathrm{Mn}$, and $\mathrm{Co}$, have one or more vacant orbitals (e.g., $d$-orbitals) that may host extra electrons. Their electronic properties need a large on-site energy $U$ to be properly understood. ${ }^{35,39,48,49}$ When extra charge carriers are (temporarily) captured by these ions, the localized charges can (i) distort local electronic band structures and (ii) introduce extra Coulomb interaction between neighboring sites. For instance, it was estimated that the interaction energy between two localized electrons on nearest neighbors can be as large as $2-3 \mathrm{eV} .{ }^{49}$ Therefore, it is conceivable that around the dopants, this type of interaction may strongly affect the dipoles (arising from displacements of ions) that exist on each unit cell of ferroelectric materials.

In addition, depending on the impurity energy $\varepsilon_{d}$ (relative to the Fermi level), the local conductivity could be changed [related to the aforementioned effect (i)]. ${ }^{50}$ If the local conductivity is high, local dipoles cannot exist because the electric field associated with dipoles will lead to the redistribution of charge carriers and eventually neutralize the bound charges induced by displacements of ions, which are responsible for forming the dipoles in the first place.
Based on the above arguments, we propose that dipoles on and around the Fe sites are suppressed and remain constant. Thus, the number of suppressed dipole should be equal to 7 times of the doped iron. The precise number could be calculated by the equation $n_{\text {dipole }}=7 \times n_{\mathrm{Fe}}$, which is always satisfied, until two or more defective dipoles contact with each other. In addition, while we aim at $\mathrm{Fe}$ doped $\mathrm{BaTiO}_{3}$, it is likely that similar arguments can be used for other transitional metals. $^{8,39,51,52}$ However, the exact number of defective dipoles induced by one dopant should be determined empirically.

We use effective Hamiltonian based MC simulations to obtain finite temperature properties. A pseudo-cubic supercell of size $12 \times 12 \times 12$ (i.e., 1728 unit cells, 8640 atoms) with periodic boundary conditions is employed in simulations. Among all the unit cells, we randomly select a certain number of them to represent Fe ion doped cells. The dipole moments on these selected sites are set to null in MC simulations. In addition, due to the influence of the Fe dopants, all its six first nearest neighbors are set to be defective too. The total energy is given by the effective Hamiltonian developed in Ref. 16

$$
\begin{aligned}
E^{\text {tot }}= & E^{\text {self }}(\{\boldsymbol{u}\})+E^{\text {dpl }}(\{\boldsymbol{u}\})+E^{\text {short }}(\{\boldsymbol{u}\}) \\
& +E^{\text {elas }}\left(\left\{\eta_{l}\right\}, \eta_{H}\right)+E^{\text {int }}\left(\{\boldsymbol{u}\},\left\{\eta_{l}\right\}, \eta_{H}\right)
\end{aligned}
$$

which consists of five parts: (i) the local-mode selfenergy, $E^{\text {self }}(\{\boldsymbol{u}\})$; (ii) the long-range dipole-dipole interaction, $E^{\mathrm{dpl}}(\{\boldsymbol{u}\})$; (iii) the short-range interaction between soft modes, $E^{\text {short }}(\{\boldsymbol{u}\})$; (iv) the elastic energy, $E^{\text {elas }}\left(\left\{\eta_{l}\right\}\right)$; and (v) the interaction between the local modes and local strain, $E^{\text {int }}\left(\{\boldsymbol{u}\},\left\{\eta_{l}\right\}\right)$, where $\boldsymbol{u}$ is the local soft-mode amplitude vector (directly proportional to the local polarization) and $\eta_{H}\left(\eta_{l}\right)$ is the sixcomponent homogeneous (inhomogeneous) strain tensor in Voigt notation. ${ }^{16}$ The parameters appearing in the effective Hamiltonian have been reported in Ref. 59.

In order to understand the doping effects, we build samples of different dopant concentrations from $0 \%$ to $2 \%$, with an increasing step of $0.2 \%$. For each of the doped samples, we gradually cool down the system from high (typically $550 \mathrm{~K}$ ) down to low (typically $30 \mathrm{~K}$ ) temperatures with a step of $10 \mathrm{~K}$. For each temperature, we typically carry out 320000 steps of MC. The first 160000 steps are used to equilibrate the system and the remaining steps to obtain averaged quantities, e.g., supercell average of local mode. For doped $\mathrm{BaTiO}_{3}$, with increasing dopant concentration, fluctuations close to phase transitions become so larger that more MC steps (e.g., 640000 ) and/or large supercells (e.g., $18 \times 18 \times 18$ ) are used to obtain satisfactory results.

To analyze dipole distributions, we have also obtained snapshots of all the dipoles in the supercell, which are typically captured at the ending stage of MC simulations when dipoles do not change much. In addition, averaged dipole configurations are obtained by storing a snapshot of dipoles every $400 \mathrm{MC}$ steps after the equilibration stage. We then use all the stored snapshots to calculate the averaged local dipole in each unit cell and perform statistics on these dipoles.

Understanding the mechanism of doping has attracted much attention. Given the variety of doping, fully understanding how doping works is an immense task and, not surprisingly, different models had been proposed. In general, depending on 
the valence state of the dopant ions, acceptor doping can induce oxygen vacancies, while donor doping will induce A-site vacancy or conduction electrons. ${ }^{7,28}$ Understanding such complex systems from numerical simulation often requires a priori assumption and modeling about doping. Proposed mechanisms include the following findings: (i) Defective dipoles can be caused by dopants (which is the mechanism we employ in this work). (ii) Local strain can be induced due to the different ionic radii of the dopants. ${ }^{29}$ (iii) As a result of oxygen vacancies and localized charges created by doping, new dipoles (defect dipoles) can be induced. ${ }^{14,42,43,53}$ (iv) Dopants can affect the grain size and/or induce new domains in the matrix material. ${ }^{9-11,47,54}$ Recently, $\mathrm{Xu}$ et al. show that antiparallel defect dipoles could induce a negative electrocaloric effect and double-peak behaviors for acceptor doped $\mathrm{BaTiO}_{3}{ }^{53}$ Cohen and co-workers successfully simulated the pinched and shifted hysteresis loop and the large recoverable electromechanical response using defect dipoles. ${ }^{13,14}$ However, due to the long range interaction of dipoles, the fixed dipoles seem to increase the phase temperature, which is not consistent with the experimental results and verified by our simulations. Therefore, further refined models or other factors, such as the one discussed here, are needed.

\section{DOPING EFFECTS}

Having described our approach, we now use MC simulations to calculate the basic properties of $\mathrm{BaTiO}_{3}$ with different dopant concentrations. We first obtain and show their hysteresis loops, polarizations, and phase transition temperatures, which are summarized to illustrate the trend of changes with respect to the dopant concentration. With all simulation results, we are also able to obtain the phase diagram of Fe-doped $\mathrm{BaTiO}_{3}$ with respect to the temperature and dopant concentration. In this way, we show that the model can indeed generate important features of $\mathrm{Fe}$-doped $\mathrm{BaTiO}_{3}$ that are observed in experiments.

\section{A. Hysteresis loop}

Figure 1 shows the hysteresis loops of the doped $\mathrm{BaTiO}_{3}$ at selected dopant concentrations $(p=0 \%, 0.6 \%, 1.2 \%$, and $1.8 \%$ ) at $300 \mathrm{~K}$. The motion of oxygen vacancies is not

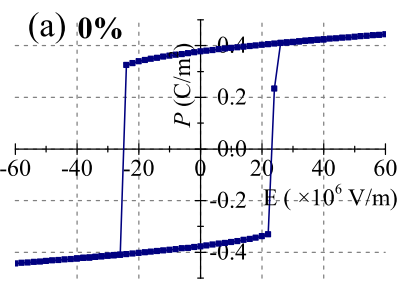

(c) $1.2 \%$
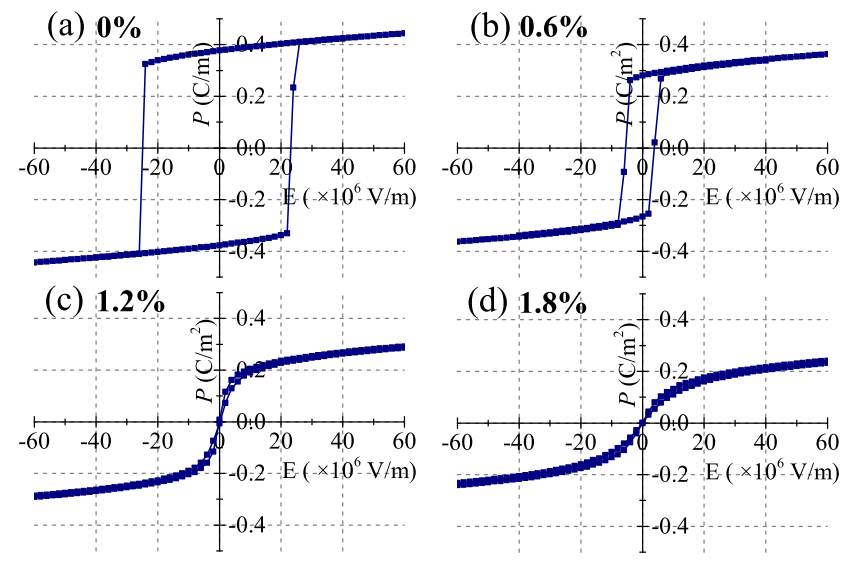

(d) $1.8 \%$

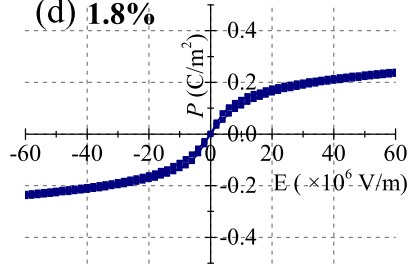

FIG. 1. [(a)-(d)] Electric hysteresis loops of doped $\mathrm{BaTiO}_{3}$ are shown for selected dopant concentrations at $300 \mathrm{~K}$. included; therefore, we are not able to observe the aging phenomenon. ${ }^{6,8,12-14,37}$ Figure 1(a) demonstrates a typical hysteresis loop of ferroelectric materials, with large coercive electric field $E_{c}$ (the electric field when polarization changes sign) and large remnant polarization $P_{r}$ (the remaining polarization when the electric field $E=0 \mathrm{~V} / \mathrm{m}$ ), as well as large saturation polarization $P_{s}$ (the polarization at very large $E$ ). As $p$ increases, both $E_{c}$ and $P_{s}$ decrease. For $p \geq 1.2 \%$, the hysteresis loop disappears completely [see Figs. 1(c) and 1(d)], indicating some critical changes that will be further discussed in Sec. V B. Such changes with respect to the dopant concentration are perhaps the most notable phenomenon observed and reported in experimental studies. ${ }^{22,40,41,55,56}$

To quantitatively check the doping effects, in Fig. 2, we plot $P_{s}$ (polarization obtained at $\left.E=6 \times 10^{7} \mathrm{~V} / \mathrm{m}\right), P_{r}$, and $E_{c}$ as functions of $p$, the dopant concentration. It can be seen that $P_{S}$ depends linearly on the concentration. According to our assumption, introducing a dopant ion will bring in seven dead dipoles near the defect sites. Therefore, at the dopant concentration $p$, the estimated saturation polarization should not exceed

$$
P_{s}(p) \simeq P_{s}(0) \times(1-7 p),
$$

which is shown as the gray dashed line in Fig. 2. As a matter of fact, $P_{S}$ declines faster than this estimation, which indicates the importance of dipole correlations in ferroelectric materials. Figure 2 also shows that both $P_{r}$ and $E_{c}$ have a sudden change at some critical values of $p(p \simeq 0.8 \%$ and $p \simeq 1.2 \%)$, beyond which they become zero.

In order to compare to experimental results, we plot the simulation results of $P_{r}$ to that of Fe-doped $0.5 \mathrm{Ba}\left(\mathrm{Zr}_{0.2} \mathrm{Ti}_{0.8}\right) \mathrm{O}_{3}-0.5\left(\mathrm{Ba}_{0.7} \mathrm{Ca}_{0.3}\right) \mathrm{TiO}_{3}{ }^{26}$ obtained experimentally in Fig. 2. The change in $P_{r}$ obtained from our simulations shows some interesting agreement with experiments. ${ }^{57}$ We note that the experimental data are normalized to compare with our simulation results. In this process, we use the polarization of the $0.25 \%$ doped sample as unit (and set this value to that obtained from our simulation) to scale the values of others.

\section{B. Phase transition}

To reveal how doped $\mathrm{BaTiO}_{3}$ evolves with temperature, we show the averaged components of the polarization $\langle\boldsymbol{P}\rangle$ in

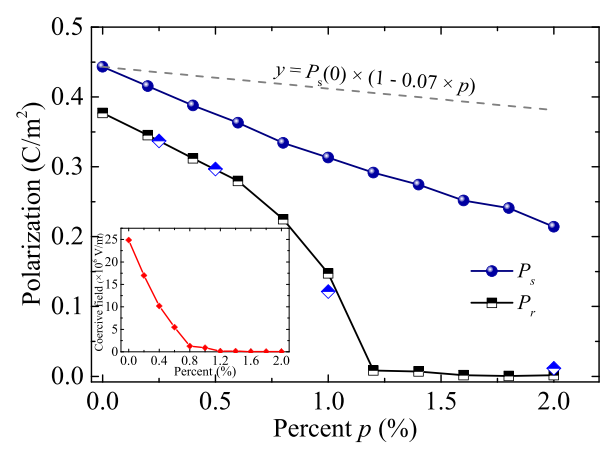

FIG. 2. The saturation polarization, $P_{s}$, the remnant polarization, $P_{r}$, and the coercive field, $E_{c}$ (inset), change with the dopant concentration (normalized experimental data from Ref. 26 are shown as half-filled blue diamonds; see the text). 
Fig. 3. For pure $\mathrm{BaTiO}_{3}$ shown in Fig. 3(a), there exist four regions:

1. For $T>390 \mathrm{~K}$, no polarization exists in any direction (i.e., $\left\langle P_{x}\right\rangle=\left\langle P_{y}\right\rangle=\left\langle P_{z}\right\rangle=0$ ), showing a paraelectric phase $(\mathrm{P})$.

2. For $200 \mathrm{~K}<T<390 \mathrm{~K},\left\langle P_{z}\right\rangle$ becomes nonzero (0.3$0.42 \mathrm{C} / \mathrm{m}^{2}$ ), showing a tetragonal phase (T, space group $P 4 \mathrm{~mm})$.

3. For $90 \mathrm{~K}<T<200 \mathrm{~K},\left\langle P_{y}\right\rangle$ and $\left\langle P_{z}\right\rangle$ are equal to each other $\left(\sim 0.35 \mathrm{C} / \mathrm{m}^{2}\right)$ while $\left\langle P_{x}\right\rangle$ is still zero, showing an orthorhombic phase (O, space group Amm2).

4. For $T<90 \mathrm{~K}$, the system reaches the rhombohedral phase $\left(\mathrm{R}\right.$, space group $R 3 m$ ) with $\left\langle P_{x}\right\rangle=\left\langle P_{y}\right\rangle=\left\langle P_{z}\right\rangle \simeq 0.3$ $\mathrm{C} / \mathrm{m}^{2}$.

We note that the above calculated results are consistent with the previous experimental ${ }^{37,55}$ and theoretical results, ${ }^{21,58,59}$ where the polarization is $0.33(\mathrm{R}), 0.36(\mathrm{O})$, and $0.27 \mathrm{C} / \mathrm{m}^{2}$ $(\mathrm{T})^{21}$ and the phase transition temperatures are $T_{2} \simeq 90 \mathrm{~K}(\mathrm{O}$ to R), $T_{1} \simeq 200 \mathrm{~K}$ (T to O), and $T_{C} \simeq 390 \mathrm{~K}$ (P to T). ${ }^{59}$

For doped $\mathrm{BaTiO}_{3}$, Figs. 3(b)-3(d) show their phase transition temperatures and phase transition sequences. At $p=0.6 \%$, all the phases appearing in pure $\mathrm{BaTiO}_{3}$ can still be seen while the phase transition temperatures are different. On the other hand, when $p=1.2 \%$, the P-T phase change happens at $T_{C} \simeq 285 \mathrm{~K}$ and the $\mathrm{T}-\mathrm{O}$ at $T_{1} \simeq 240 \mathrm{~K}$, leaving a narrower region for the $\mathrm{T}$ phase. Finally, at $T_{2}=180 \mathrm{~K}$, the system changes from the Amm2 phase (orthorhombic) to the $\mathrm{Cm}$ phase (monoclinic, $\mathrm{M}$ ). At $p=1.8 \%$, the phase transitions become completely different with the $\mathrm{Cm}$ phase appearing at the lowest temperatures. In general, it can be seen from Fig. 3 that $\langle\boldsymbol{P}\rangle$ decreases with $p$. In addition, for large $p$, we have to endure some ambiguity in determining the phase transition temperatures as the phase transition becomes diffused.

Doping effects on phase transitions can be summarized in the phase diagram shown in Fig. 4, which reveals that for $\mathrm{Fe}$-doped $\mathrm{BaTiO}_{3}$ with $p \leq 2.0 \%$, four phases exist: (i) between $T_{C}$ and $T_{1}$, the $P 4 m m$ phase; (ii) between $T_{1}$ and $T_{2}$, the $A m m 2$ phase; (iii) below $T_{2}$, the $R 3 m$ or $C m$ phase. The existence of the $R 3 \mathrm{~m}$ and the $\mathrm{Cm}$ phase indicates a possible morphotropic boundary that is interesting for potential performance enhancement. ${ }^{60}$ We can also see that, as $p$ increases,

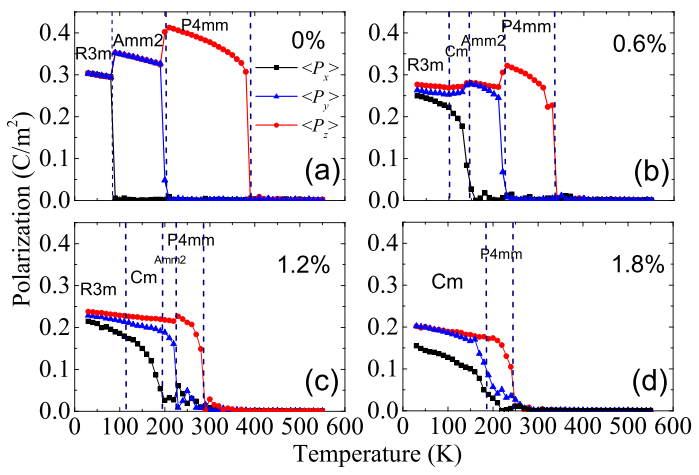

FIG. 3. The polarization versus temperature plots are shown for selected dopant concentrations. The vertical dashed lines show the separation between different phases.

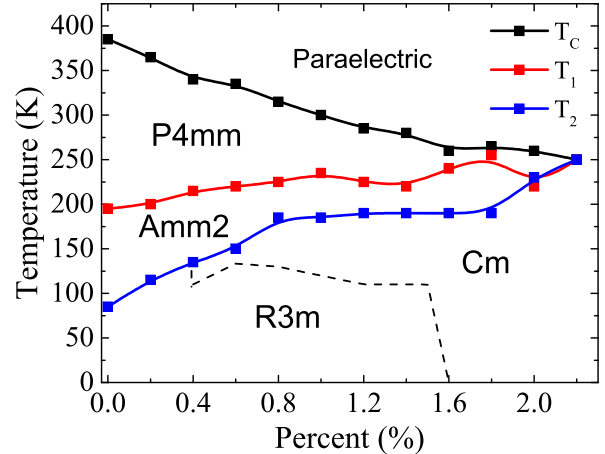

FIG. 4. Phase transition temperatures are plotted against dopant concentrations.

$T_{C}$ decreases quickly from $375 \mathrm{~K}$ (at $p=0.0 \%$ ) to $\sim 250 \mathrm{~K}$ (at $p=2.0 \%$ ), resulting in a decreasing rate of $57 \mathrm{~K} /(1 \%$ doping). Such a decreasing rate is much smaller for Mn (with experimental results being $\sim 15 \mathrm{~K} /(1 \% \text { doping })^{39}$ and other similar elements. ${ }^{51,52}$ On the other hand, $T_{2}$ increases from $85 \mathrm{~K}($ at $p=0 \%)$ to $\sim 250 \mathrm{~K}$ (at $p=2.0 \%$ ). Moreover, the shift of $T_{1}$ is relatively small compared to the other two. The convergence of $T_{C}, T_{1}$, and $T_{2}$ around $p \simeq 2.0 \%$ agrees very well with the experimental results with doped $\mathrm{BaTiO}_{3},{ }^{51}$ which also slightly resembles what happens with $\mathrm{Ba}(\mathrm{Zr}, \mathrm{Ti}) \mathrm{O}_{3}$ when $\mathrm{Zr}$ concentration increases..$^{22,61,62}$ Both the variation trend and speed of characteristic temperature will be further discussed in Sec. V C.

\section{DIPOLE DISTRIBUTION}

In order to understand doping effects on the macroscopic properties of $\mathrm{BaTiO}_{3}$, we need to know how microscopic dipole configuration responds to doping. In this section, we focus on orientation and magnitude distributions of dipoles in pure and doped $\mathrm{BaTiO}_{3}$ to gain insights into how doping works.

\section{A. Orientation}

In Fig. 5, the orientation distribution of dipoles for pure and $0.6 \%$ doped $\mathrm{BaTiO}_{3}$ is shown for selected temperatures $(450,300,170$, and $30 \mathrm{~K})$. At each temperature, we categorize dipoles as $\langle 100\rangle,\langle 110\rangle$, or $\langle 111\rangle$, depending on their orientations. Figure 5(i) shows a schematic drawing of how the categorization is performed, and Figs. 5(a)-5(h) show the number of dipoles in each category for pure and doped $\mathrm{BaTiO}_{3}$ at the selected temperatures.

Figure 5(a) shows that, at $T=450 \mathrm{~K}$ (paraelectric phase), owing to the large thermal fluctuation, all three types have a significant number of dipoles, with $20.2 \%$ for $\langle 100\rangle, 46.6 \%$ for $\langle 110\rangle$, and $33.2 \%$ for $\langle 111\rangle$. The $0.6 \%$ doped $\mathrm{BaTiO}_{3}$ [Fig. 5(e)] almost has the same distribution. Given such a distribution, at this temperature, the averaged values (e.g., $\langle\boldsymbol{P}\rangle)$ are very small (see Fig. 3), producing a paraelectric phase.

At $T=300 \mathrm{~K}$ (macroscopic $P 4 m m$ phase), Fig. 5(b) shows that the number of $\langle 100\rangle$ dipoles has significantly increased, while the number in the other two categories decreased. Besides, snapshots of dipole configurations reveal that most 

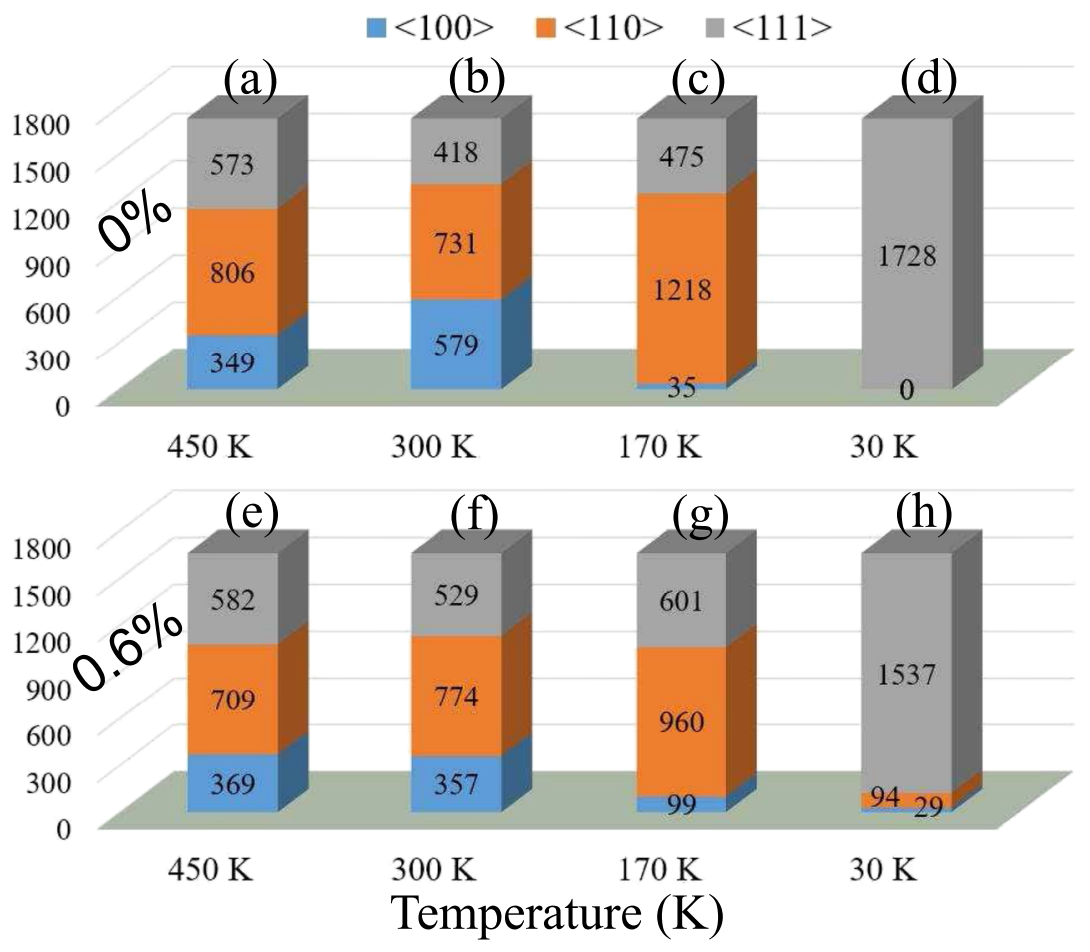

FIG. 5. Dipole orientation distribution calculated from dipole snapshots at $450 \mathrm{~K}, 300 \mathrm{~K}, 170 \mathrm{~K}$, and $30 \mathrm{~K}$. [(a)-(d)] Pure $\mathrm{BaTiO}_{3}$; [(e)-(h)] doped $\mathrm{BaTiO}_{3}$ with $p=0.6 \%$; and (i) dipole orientation distribution on a unit sphere.
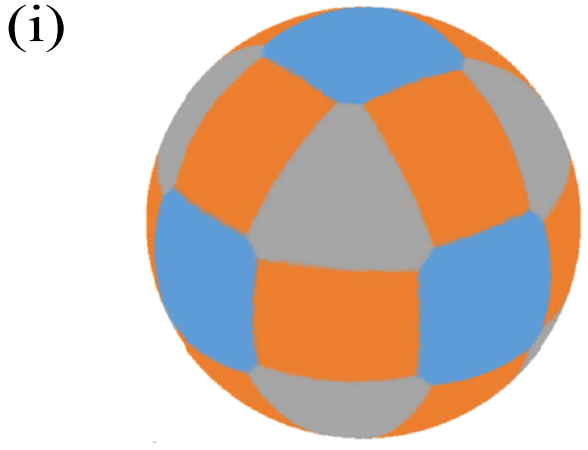

dipoles ( $>95 \%$ ) have positive $z$ components for both pure and $0.6 \%$ doped $\mathrm{BaTiO}_{3}$. This means that a preferred direction ([001] here) has been established at $300 \mathrm{~K}$ in the system, likely due to the long-range dipole-dipole interaction. At $T$ $=170 \mathrm{~K}[$ Amm2, see Figs. 5(c) and 5(g)], the averaged polarization $\langle\boldsymbol{P}\rangle$ is along the [011] direction, consistent with the experimental results. However, for a single snapshot, a large portion $(\sim 27 \%)$ of dipoles belongs to the $\langle 111\rangle$ category. In addition, doping gives rise to more "disobedient" dipoles that do not follow the overall orientation. For instance, in pure $\mathrm{BaTiO}_{3}, 70.5 \%$ of the dipoles belong to the $\langle 110\rangle$ category, while in the $0.6 \%$ doped $\mathrm{BaTiO}_{3}$, the number of dipoles is smaller (57.8\%). At $T=30 \mathrm{~K}$ ( $R 3 \mathrm{~m}$ phase), Fig. 5(d) shows that all the dipoles in $\mathrm{BaTiO}_{3}$ belong to the $\langle 111\rangle$ category. In fact, all the dipoles point to a particular one of the $\langle 111\rangle$ directions, making the system the $R 3 m$ phase. In contrast, the $0.6 \%$ doped $\mathrm{BaTiO}_{3}$ again have some "disobedient" dipoles belonging to the other two types [Fig. 5(h)]. In general, Fig. 5 shows that the number of dominant dipoles decreases with doping.

Below $T_{C}$, the system includes three phases, $R 3 m(\mathrm{Cm})$, $A m m 2$, and $P 4 m m$, with the average polarization $(\langle\boldsymbol{P}\rangle)$ pointing along $\langle 111\rangle,\langle 110\rangle$, and $\langle 100\rangle$, respectively. The local dipole structure information associated with Fig. 5 provides some insights about the phase transitions shown in Figs. 3 and 4. It can be deduced as follows: (i) The $\mathrm{P}$ to $\mathrm{T}$ phase transition is mostly an order-disorder phase transition since the local dipole orientation distribution remains similar (i.e., no major dipole rotation happens) while a ferroelectric phase establishes when the temperature drops from $450 \mathrm{~K}$ to $300 \mathrm{~K}$. Therefore the macroscopic phase transition happens mostly due to the increase in the correlation length of local dipoles. (ii) The T to $\mathrm{O}$ and $\mathrm{O}$ to $\mathrm{R}$ phase transitions are mixtures of displacive and order-disorder type. The complementary changes in the $\langle 100\rangle$ and $\langle 110\rangle$ dipoles mark the orientation conversion from the $\langle 100\rangle$ type to the $\langle 110\rangle$ type dipoles (for $\mathrm{O}$ to $\mathrm{R}$, it is the $\langle 110\rangle$ to $\langle 111\rangle$ conversion). At the same time, the presence of the $\langle 111\rangle$ dipoles in all four temperatures indicates the existence of uncorrelated local rhombohedral regions, which eventually become correlated (via order-disorder phase transition) at low temperatures to form the long-range rhombohedral phase [see Figs. 5(c), 5(d), 5(g), and 5(h)]. Such observations are critical to understand how $T_{C}, T_{1}$, and $T_{2}$ change with respect to doping. 


\section{B. Magnitude}

Since the distribution of dipole components $\left(u_{x}, u_{y}\right.$, and $u_{z}$ ) can shed more light on how doping works, we will also analyze the distributions of dipole magnitudes at different temperatures and pay particular attention to their evolution with dopant concentration.

Figure 6(a) compares the distributions of pure $\mathrm{BaTiO}_{3}$ to those of the $0.4 \%$ doped one. At $450 \mathrm{~K}$, both samples have nearly symmetric distribution for $u_{x, y, z}$ with respect to $\boldsymbol{u}=0$, which results in the paraelectric phase $(\langle\boldsymbol{u}\rangle=0)$ when averaging is performed. At $300 \mathrm{~K}$, for both samples, $u_{z}$ deviates from the symmetric distribution around zero and centers around 0.038. Owing to this deviation, the averaged local mode $\left\langle u_{z}\right\rangle$ is no longer zero and the sample displays a [001] polarization (T phase). Careful inspection shows that $u_{z}$ of the $0.4 \%$ doped $\mathrm{BaTiO}_{3}$ has a slightly broader peak, which is shifted toward 0 (centering around 0.032). Similar phenomena are also observed at $170 \mathrm{~K}$ and $30 \mathrm{~K}$. In general, compared to pure $\mathrm{BaTiO}_{3}$, the $0.4 \%$ sample has its characteristic peak slightly modified, becoming broader and lower, and its center moving towards zero. It can be concluded that a small amount of "disobedient" dipoles must exist that have slightly changed these distributions. These "disobedient" dipoles can also be used to account for the increased number of minority dipoles seen in Figs. 5(e)-5(h). Such dipoles will be further discussed in Sec. V A. Moreover, we note that the results for pure $\mathrm{BaTiO}_{3}$ presented here are consistent with Ref. 21.

More details are shown in Figs. 6(b) and 6(c). At $300 \mathrm{~K}$ [Fig. 6(b)], the distribution of $u_{z}$ changes strongly with doping, following the aforementioned trend. At $p=1.0 \%$, the peak is already lower by $\sim 50 \%$ than the initial peak and the center of the peak has changed from $\sim 0.038$ to $\sim 0.02$. When $p=1.2 \%$, the distribution almost becomes symmetric around zero, giving rise to a paraelectric phase, which is consistent with Fig. 3(c). At $170 \mathrm{~K}$ [Fig. 6(c)], while $u_{x, y}$ have peaks of nonzero values, $u_{z}$ starts from a symmetric distribution around zero and develops a peak centering around 0.018 at $p=0.8 \%$ (which changes the system from the Amm2 to the $\mathrm{Cm}$ phase), but the peak shifts toward zero upon further increase of doping concentration. This feature is reflected by the Amm 2 and $\mathrm{Cm}$ phase boundaries shown in Fig. 4.

\section{UNDERSTANDING DOPING EFFECTS}

In order to identify the mechanism of doping, we also examine how dipole configurations evolve during MC simulations. Our results reveal that, in doped $\mathrm{BaTiO}_{3}$, some dipoles become more active and tend to change a lot during simulations even far away from the phase transition temperature. In this section, we will first introduce the concept of "active dipole"

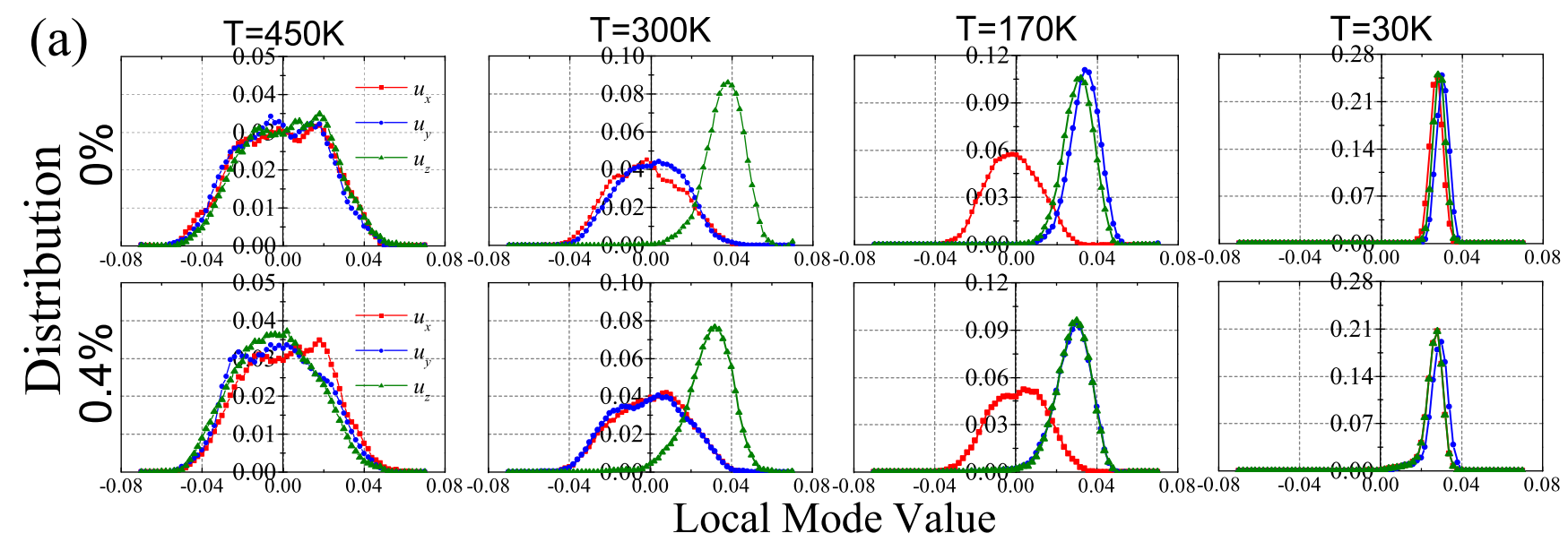

(b)

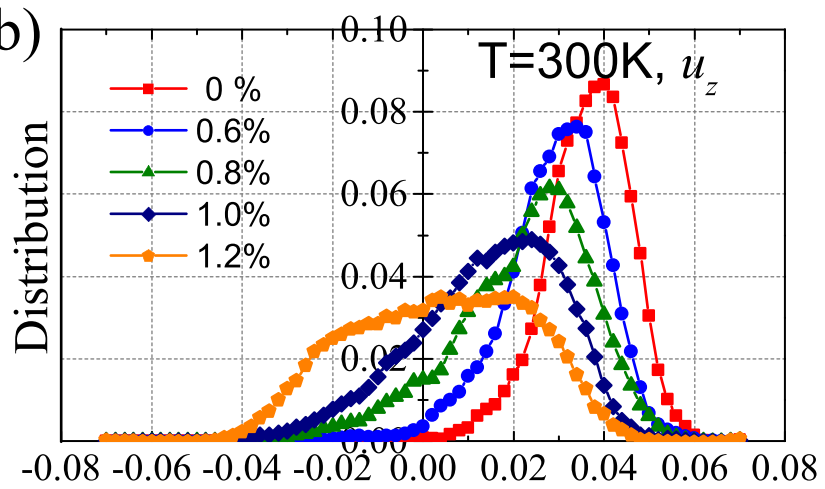

Local Mode Value

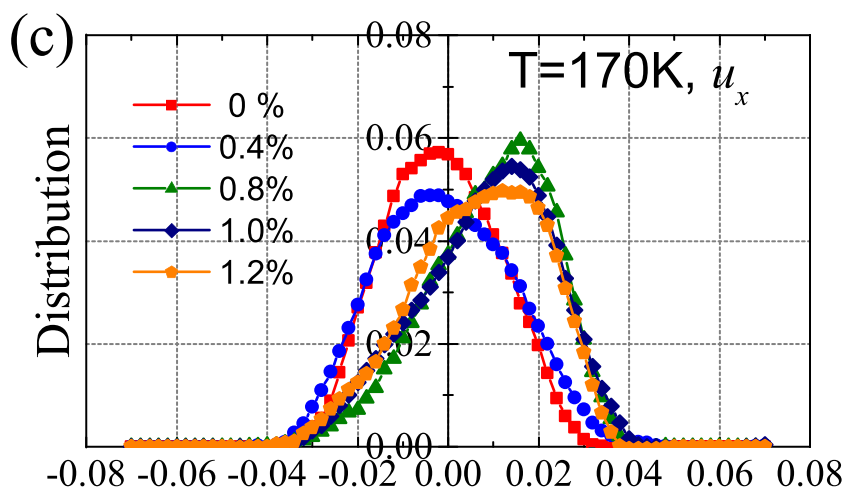

Local Mode Value

FIG. 6. (a) The distributions of dipoles for pure $\mathrm{BaTiO}_{3}$ (first row) and $0.4 \%$ doped $\mathrm{BaTiO}_{3}$ (second row) at selected temperatures. (b) $u_{z}$ distribution for different doping concentrations at $300 \mathrm{~K}$. (c) $u_{x}$ distribution for different doping concentrations at $170 \mathrm{~K}$. All dipole distributions are normalized. 
and then use it along with dipole distributions to understand doping effects shown in Sec. III.

\section{A. Active dipoles}

Active dipoles are those dipoles that can substantially change its states during MC simulations. Unlike the majority of dipoles, which often fluctuate around their equilibrium position and determine the macroscopic polarization, active dipoles can easily change in direction and magnitude, while such rotations are forbidden for most dipoles. In other words, the most important quality of active dipoles is that they are much more active than other dipoles even at temperatures much lower than the phase transition temperature.

Practically, we identify active dipoles with the following procedure: (i) We find the average polarization $(\langle\boldsymbol{P}\rangle)$ at a given temperature. (ii) Choose a snapshot from the $\mathrm{MC}$ simulation and find dipoles pointing along directions that are different from $\langle\boldsymbol{P}\rangle$. According to our simulation, these dipoles are special in that (i) they have relatively fixed locations, (ii) their quantity is very small (less than $3.3 \%$ for Fe-doping $\mathrm{BaTiO}_{3}$ at $p=2.0 \%$ ), and (iii) their directions change from time to time, making experimental detection hard.

Figure 7(a) shows the number of active dipoles as a function of temperature, which shows that even below the phase transition temperature, the number of active dipoles is not exactly zero. For instance, at $100 \mathrm{~K}$, the BTO has the macroscopic $R 3 m$ phase with most of the dipoles pointing along, e.g., the [111] direction. However, there are a few of the dipoles [see the results labeled as $u_{x}$-active at $100 \mathrm{~K}$ in Fig. 7(a)] that are determined to point to the $[\overline{1} 11]$ direction-those dipoles are the active dipoles. As the temperature reaches $200 \mathrm{~K}$, half of the dipoles become $u_{x}$-active, making the system an Amm2 phase $\left(\left\langle P_{x}\right\rangle \simeq 0,\left\langle P_{y}\right\rangle=\left\langle P_{z}\right\rangle \neq 0\right)$; in such a case, most dipoles are along the [111] and $[\overline{1} 11]$ directions. However, there are dipoles pointing along, e.g., $[1 \overline{1} 1]$, which are the active dipoles [see the results labeled as $u_{y}$-active at $200 \mathrm{~K}$ in Fig. 7(a)]. At $300 \mathrm{~K}$, both the $u_{x}$-active and $u_{y}$-active dipoles occupy half of the dipole population, making the system a $P 4 m m$ phase with only $\left\langle P_{z}\right\rangle$ being nonzero. While most dipoles belong to the set of four directions ([111], $[\overline{1} 11],[1 \overline{1} 1]$, and $[\overline{1} \overline{1} 1])$, the active dipoles have different directions (e.g., $[11 \overline{1}]$ ) [see the results labeled as $u_{x}$-active at $200 \mathrm{~K}$ in Fig. 7(a)].

When the temperature increases, the number of active dipoles initially increases slowly at low temperatures, and then at certain temperatures, it suddenly increases, finally reaching half of the whole dipole population. Compared to Fig. 3(b), it is important to note the large jumps can be associated with the phase change temperatures $T_{2}, T_{1}$, and $T_{C}$. We also find that doped $\mathrm{BaTiO}_{3}$ have bridging points [circled points in Fig. 7(a)] that are hard to find in pure $\mathrm{BaTiO}_{3}$. The existence of such points moderate the phase transition, causing more diffusive transition peaks observed in experiments. ${ }^{26,54}$

It is also critical to know how the number of active dipoles depends on the dopant concentration. Figure 7(b) reveals that the number of active dipoles (red arrows) increases with dopant concentration. More importantly, Fig. 7(b) shows the proximity of active dipoles to defective dipoles, indicating a close relation between them.

The origin of active dipoles can be understood in terms of local chemistry and the dipole vacuum associated with defective dipoles since (i) Fe ions can be taken as negative when surrounded by $\mathrm{Ti}^{4+}$ ions (see Fig. 8) from valence bond theory ${ }^{63,64}$ and (ii) the dipole vacuum can induce bound charges. ${ }^{65}$ These two factors inevitably affect the local electric field, making it different from the overall spontaneous internal electric field, which in turn creates active dipoles around doping sites and distorts dipole distributions.

\section{B. Hysteresis loop}

Figure 2 has shown that the saturation polarization, $P_{s}$, decreases with dopant concentration, which can be understood (a)

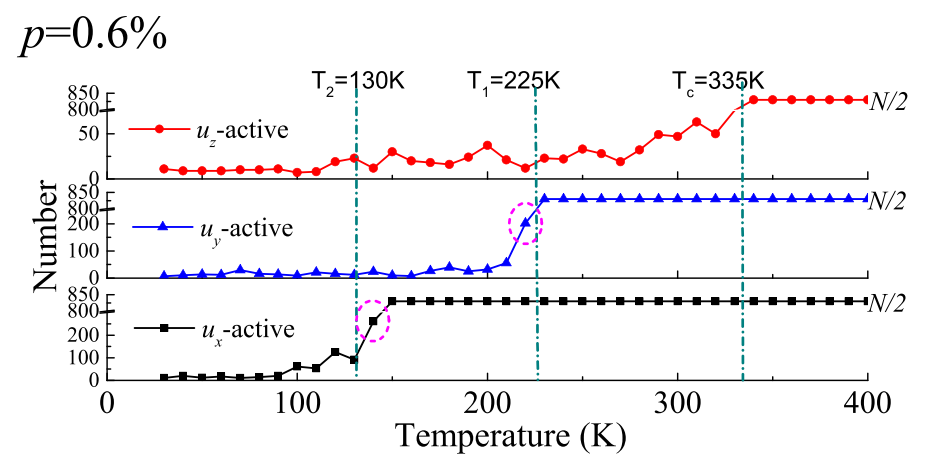

(b)
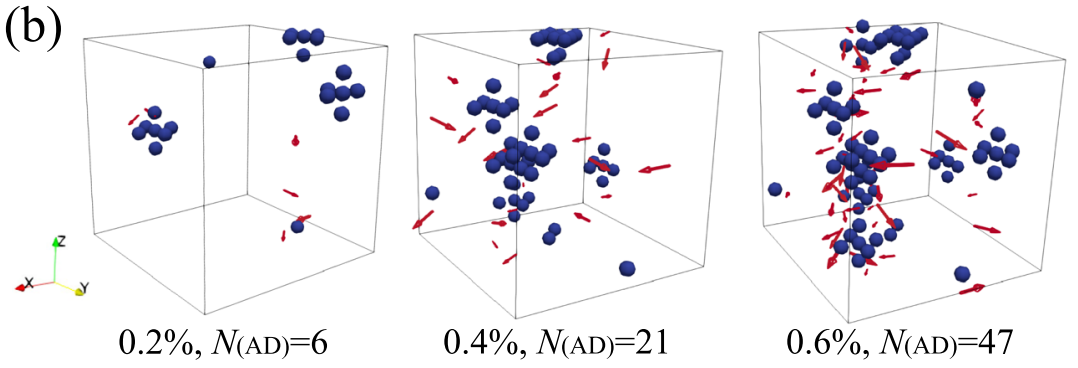

FIG. 7. (a) The evolution of active dipoles for $0.6 \% \mathrm{BaTiO}_{3}$, and (b) the locations and numbers of active dipoles (red arrows) and defective dipoles (blue balls) at $300 \mathrm{~K}$ for $p=0.2 \%, 0.4 \%$, and $0.6 \%$. The three curves in (a) indicate the number of active dipoles that have opposite direction to $\left\langle P_{x}\right\rangle,\left\langle P_{y}\right\rangle$, or $\left\langle P_{z}\right\rangle . N_{(A D)}$ means the number of active dipoles. The circled points indicate notable bridging temperatures between different phases which are caused by doping. 


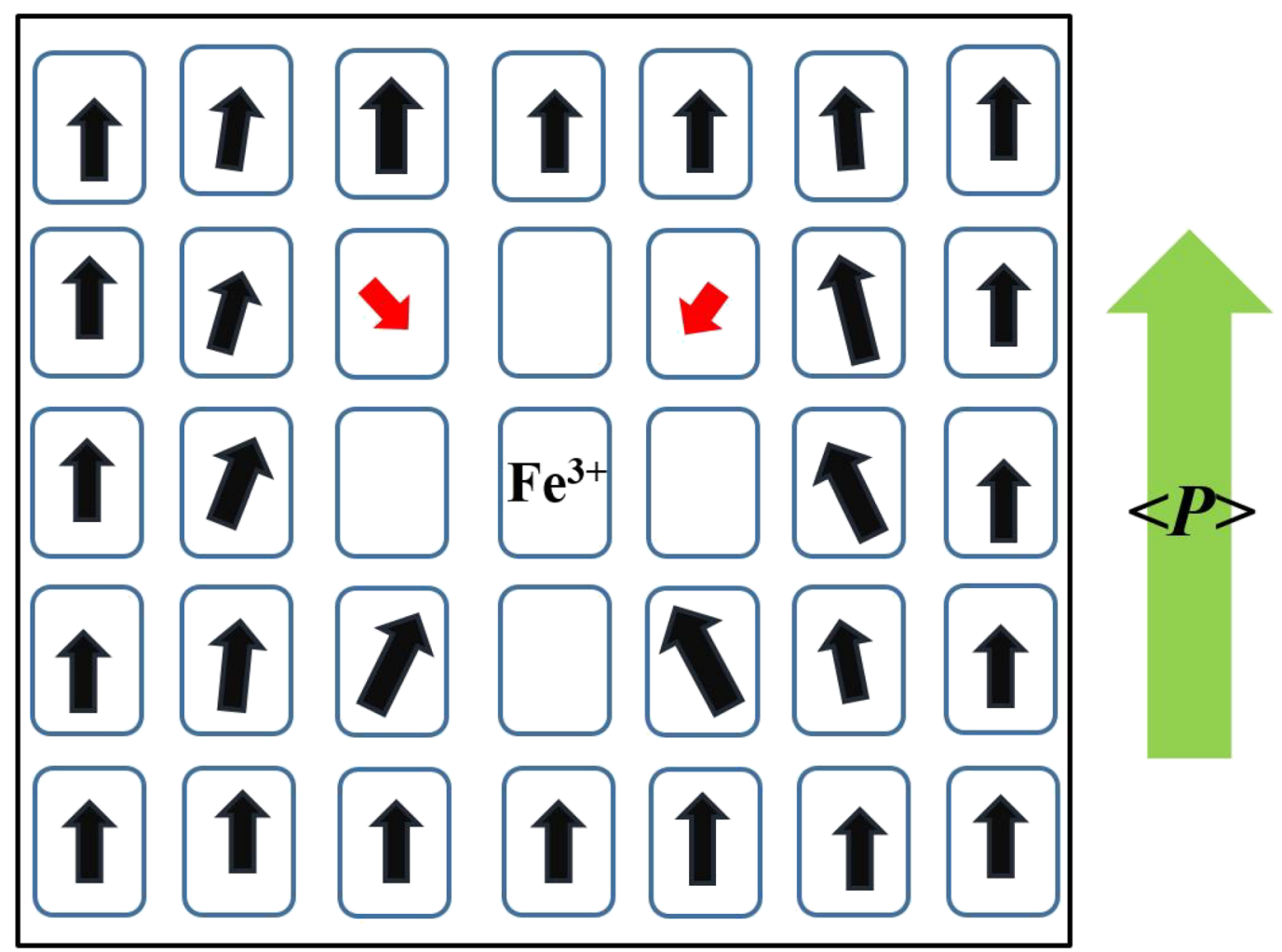

FIG. 8. Schematic drawing for the origin of active dipoles.

with the following considerations: (i) Dopant ions decrease the total number of dipoles. (ii) Dopant ions also give rise to active dipoles that modify the dipole distribution of pure $\mathrm{BaTiO}_{3}$ (see Sec. IV and Fig. 6). (iii) The long-range dipoledipole interaction is disrupted by the defective dipoles and the induced active dipoles so that dipoles are not aligned as well as those in pure $\mathrm{BaTiO}_{3}$. Owing to these factors, $P_{s}$ decreases with dopant concentration.

The remnant polarization, $P_{r}$, also shows decreasing tendency with doping. As a matter of fact, doping induces more active dipoles, causing distribution variation as shown in Fig. 6(b). Such more symmetric distribution reduces the internal electric field, not surprisingly, causing $P_{r}$ to decrease. The loss of $P_{r}$ at $p \simeq 1.2 \%$ (Fig. 2) is consistent with the distribution variation shown in Fig. 6(b), where doping makes the distribution peak shifts toward $u_{z}=0$, eventually becoming a symmetric distribution at $p \simeq 1.2 \%$ where no net internal electric field is present to support $P_{r}$.

The change of the coercive field $E_{c}$ can be understood in a similar way. As the dipole distribution becomes increasingly symmetric, the internal electric field (associated with dipoles) becomes weaker. Therefore a smaller external electric field can reverse the polarization, starting from the active dipoles and eventually causing an avalanche change in the dipole direction.

Moreover, circled points in Fig. 7(b) indicate that doped $\mathrm{BaTiO}_{3}$ can have gradual changes of polarization with temperature due to the increased number of active dipoles, which effectively reduce the sharpness of phase transitions (see Fig. 3), causing the gradual disappearance of first-order transitions (in favor of second order phase transitions).

\section{Phase transition temperature}

In order to understand the variation of $T_{C}, T_{1}$, and $T_{2}$ with doping, we first note that there are two types of phase transitions for pure and doped $\mathrm{BaTiO}_{3}$ : order-disorder and displacive. The order-disorder phase transition happens when the correlation length between local dipoles becomes significantly large. On the other hand, the displacive phase transition is related to rotations of long-range ordered dipoles. These two types of phase transitions have been discussed in Sec. IV A. In addition, it is important to note that doping in $\mathrm{BaTiO}_{3}$ favors the $\langle 111\rangle$ and $\langle 110\rangle$ dipoles when the internal electric field becomes weaker, ${ }^{66}$ which can be seen by comparing Fig. 5(f) with Fig. 5(b), which also shows that, with $0.6 \%$ doping, the number of $\langle 111\rangle$ dipoles has risen to more than $31 \%$ of all dipoles, much higher than the pure $\mathrm{BaTiO}_{3}$.

Since doping introduces defective dipoles and active dipoles that hinder the establishment of long range correlation in doped $\mathrm{BaTiO}_{3}$, lower $T_{C}$ is thus necessary to overcome such perturbation to enable the order-disorder phase transition from the paraelectric phase to the ferroelectric $\mathrm{T}$ phase. In addition, in the $\mathrm{T}$ phase, doping makes the $u_{z}$ distribution broader and more symmetric [see Fig. 6(b)], which shows that the $\mathrm{P}$ to $\mathrm{T}$ phase transition can be less dramatic, explaining the diffused phase transition and diffusive dielectric peak seen in experiments. ${ }^{25,26,29,54}$

On the other hand, $T_{2}$ and $T_{1}$ do not decrease with doping (in fact $T_{2}$ increases) because (i) the associated phase transitions (e.g., the $\mathrm{O}$ to $\mathrm{R}$ phase transition) is mostly a displacive phase transition (long range order has already been 
established), i.e., dipoles need to rotate to change from the $A m m 2$ phase to the $R 3 m$ (or $\mathrm{Cm}$ ) phase [see Figs. 5(c), 5(d), $5(\mathrm{~g})$, and $5(\mathrm{~h})]$, and (ii) doping favors the $\langle 111\rangle$ dipoles that are building blocks of the $\mathrm{R} 3 \mathrm{~m}$ or the $\mathrm{Cm}$ phases. Therefore, in this phase transition, the influence of doping on the formation of long-range ordering is less relevant, while the favored $\langle 111\rangle$ dipoles help the displacive phase transition to happen, making $T_{2}$ increase with doping. The fate of $T_{1}$ can be similarly understood by considering the relative importance of the two types of phase transitions.

From our simulations, we know that the number of defective dipoles caused by doping plays an important role in the variation of the phase transition temperature with dopant concentration. Interestingly, different dopants have different abilities to induce defective dipoles, largely depending on their $3 d$ electrons. For instance, $\mathrm{Mn}$-doped $\mathrm{BaTiO}_{3}$ has less defective dipoles (compared to $\mathrm{Fe}$ doping) since both $\mathrm{Mn}^{4+}$ and $\mathrm{Mn}^{3+}$ can exist in the system and $\mathrm{Mn}^{4+}$ is compatible with $\mathrm{Ti}^{4+}$ in terms of charge state. ${ }^{39,51}$ Therefore, $\mathrm{Mn}$-doped $\mathrm{BaTiO}_{3}$ shows less dramatic variation in the phase transition temperature, which is consistent with the experimental results. ${ }^{39,51}$

\section{CONCLUSION}

In this work, we have developed a computationally tractable model, which resolves around defective dipoles, to help understanding experimental results with doped ferroelectrics. This empirical model can successfully reproduce many important experimental results, including the ferroelectric hysteresis loop, the phase transition temperature, and their variation with doping. Based on the simulation results, we propose the existence of active dipoles and show their influence on the dipole distributions, which in turn can account for the experimentally observed phenomena. With this approach, we are also able to correlate microscopic dipole structural features with macroscopic phenomena. In addition, we believe that other interpretations (e.g., defect dipole, oxygen vacancy, and local strain) also need to invoke defective dipoles before they can explain macroscopic ferroelectric properties. Therefore, the creation of defective dipoles (as well as active dipoles) may be seen as a universal mechanism to account for effects associated with minuscule doping. We thus hope that this study will help understanding and designing novel doped perovskites to achieve desired material performance.

\section{ACKNOWLEDGMENTS}

We thank P. Rinke from Aalto University for fruitful discussion. This work was financially supported by the National Natural Science Foundation of China (NSFC) (Grant Nos. 11574246, 51390472, and U1537210) and National Basic Research Program of China (Grant No. 2015CB654903). We also acknowledge the "111 Project" of China (Grant No. B14040). L.J. acknowledges NSFC (Grant No. 51772239) and the Fundamental Research Funds for the Central Universities (XJTU). Z.J. acknowledges NSFC (Grant No. 11804138) and China Postdoctoral Science Foundation (Grant No. 2018M641905). L.L. acknowledges NSFC (Grant No. 11564010) and the Natural Science Foundation of Guangxi
(Grant Nos. GA139008 and AA138162). D.W. also thanks the support from the China Scholarship Council (Grant No. 201706285020).

${ }^{1}$ Y. S. Jung, E. S. Na, U. Paik, J. Lee, and J. Kim, Mater. Res. Bull. 37, 1633 (2002).

${ }^{2}$ C. H. Yang, J. Seidel, S. Y. Kim, P. B. Rossen, P. Yu, M. Gajek, Y. H. Chu, L. W. Martin, M. B. Holcomb, Q. He, P. Maksymovych, N. Balke, S. V. Kalinin, A. P. Baddorf, S. R. Basu, M. L. Scullin, and R. Ramesh, Nat. Mater. 8, 485 (2009).

${ }^{3}$ T. Shi, G. Li, and J. Zhu, Ceram. Int. 43, 2910 (2017).

${ }^{4}$ A. Limpichaipanit and A. Ngamjarurojana, Ceram. Int. 43, 4450 (2017).

${ }^{5}$ C. Ang, Z. Yu, and L. E. Cross, Phys. Rev. B 62, 228 (2000).

${ }^{6}$ X. B. Ren, Nat. Mater. 3, 91 (2004).

${ }^{7}$ G. C. Deng, G. R. Li, A. Ding, and Q. R. Yin, Appl. Phys. Lett. 87, 192905 (2005).

${ }^{8}$ L. Zhang and X. Ren, Phys. Rev. B 73, 094121 (2006).

${ }^{9}$ L. Hong, A. K. Soh, and Q. G. Du, and J. Y. Li, Phys. Rev. B 77, 094104 (2008).

${ }^{10}$ L. Jin, Z. B. He, and D. Damjanovic, Appl. Phys. Lett. 95, 012905 (2009).

${ }^{11}$ L. Jin, V. Porokhonskyy, and D. Damjanovic, Appl. Phys. Lett. 96, 242902 (2010).

${ }^{12}$ P. Gao, C. T. Nelson, and J. R. Jokisaari, Nat. Commun. 2, 591 (2011).

${ }^{13}$ J. B. J. Chapman, R. E. Cohen, A. V. Kimmel, and D. M. Duffy, Phys. Rev. Lett. 119, 177602 (2017).

${ }^{14}$ S. Liu and R. E. Cohen, Appl. Phys. Lett. 111, 082903 (2017).

${ }^{15}$ One goal of experimental work is usually to find the origin or cause, $c$, given the phenomena or evidence, $E$, observed in an experiment. If there are several candidates as possible causes $\left(c_{i}\right.$ where $\left.i=1,2,3, \ldots\right)$, we often implicitly evaluate the function $f(E)=\operatorname{argmax} P\left(c_{i} \mid E\right)$, where $P\left(c_{i} \mid E\right)$ is the conditional probability that provides the likelihood of $c_{i}$ given $E$, in order to suggest the most likely cause of observed experimental phenomena. The calculation of $P\left(c_{i} \mid E\right)$ is usually a difficult task. One way to address this problem is to use Bayes' theorem, which states that $P\left(c_{i} \mid E\right)=P\left(E \mid c_{i}\right) P\left(c_{i}\right) / P(E)$. Therefore the aforementioned equation can be converted to $f(E)=\underset{c_{i}}{\operatorname{argmax}} P\left(E \mid c_{i}\right) P\left(c_{i}\right)$, where $P(E)$ in the denominator is removed since only argmax is needed. The above two expressions sometimes are called discriminative and generative approaches in machine learning. In this work, we show, among other things, that $P\left(E \mid c_{0}\right)$ is very large where $c_{0}$ represents one particular cause, i.e., doping can induce defective dipoles.

${ }^{16}$ W. Zhong, D. Vanderbilt, and K. M. Rabe, Phys. Rev. B 52, 6301 (1995).

${ }^{17}$ D. Wang, J. Hlinka, A. A. Bokov, Z. G. Ye, P. Ondrejkovic, J. Petzelt, and L. Bellaiche, Nat. Commum. 5, 5100 (2014).

${ }^{18}$ A. R. Akbarzadeh, S. Prosandeev, J. E. Walter, A. Al-Barakaty, and L. Bellaiche, Phys. Rev. B 91, 214117 (2015).

${ }^{19}$ D. Wang, A. A. Bokov, Z. G. Ye, J. Hlinka, and L. Bellaiche, Nat. Commun. 7, 11014 (2016).

${ }^{20}$ M. S. Senn, D. A. Keen, T. C. A. Lucas, J. A. Hriljac, and A. L. Goodwin, Phys. Rev. Lett. 116, 207602 (2016).

${ }^{21}$ Y. Qi, S. Liu, I. Grinberg, and A. M. Rappe, Phys. Rev. B 94, 134308 (2016).

${ }^{22}$ Z. Yu, C. Ang, R. Y. Guo, and A. S. Bhalla, J. Appl. Phys. 92, 1489 (2002).

${ }^{23}$ A. R. West, T. B. Adams, F. D. Morrison, and D. C. Sinclair, J. Eur. Ceram. Soc. 24, 1439 (2004).

${ }^{24}$ B. Xu, K. B. Yin, J. Lin, Y. D. Xia, X. G. Wan, J. Yin, X. J. Bai, J. Du, and Z. G. Liu, Phys. Rev. B 79, 134109 (2009).

${ }^{25}$ D. Hennings, A. Schnell, and G. Simon, J. Am. Ceram. Soc. 65, 539 (1982).

${ }^{26}$ L. Jin, R. J. Huo, R. Guo, F. Li, D. Wang, Y. Tian, Q. Hu, X. Y. Wei, Z. He, Y. Yan, and G. Liu, ACS Appl. Mater. Interfaces 8, 31109 (2016).

${ }^{27}$ S. Yasmin, S. Choudhury, M. A. Hakim, A. H. Bhuiyan, and M. J. Rahman, J. Mater. Sci. Technol. 27, 759 (2011).

${ }^{28}$ M. Ganguly, S. K. Rout, T. P. Sinha, S. K. Sharma, H. Y. Park, C. W. Ahn, and I. W. Kim, J. Alloys Compd. 579, 473 (2013).

${ }^{29}$ T. Chakraborty and S. Ray, J. Alloy. Compd. 610, 271 (2014).

${ }^{30}$ U. Weber, G. Greuel, U. Boettger, S. Weber, D. Hennings, and R. Waser, J. Am. Ceram. Soc. 84, 759 (2001).

${ }^{31}$ N. Baskaran and H. Chang, J. Mater. Sci.: Mater. Electron. 12, 527 (2001).

${ }^{32}$ Y. Wang and C. W. Nan, Appl. Phys. Lett. 89, 052903 (2006).

${ }^{33}$ N. Nanakorn, P. Jalupoom, N. Vaneesorn, and A. Thanaboonsombut, Ceram. Int. 34, 779 (2008).

${ }^{34}$ T. Kundu, A. Jana, and P. Barik, Bull. Mater. Sci. 31, 501 (2008).

${ }^{35}$ S. O. Leontsev and R. E. Eitel, J. Am. Ceram. Soc. 92, 2957 (2009). 
${ }^{36}$ Z. Guo, L. Yang, H. Qiu, X. Zhan, J. Yin, and L. Cao, Mod. Phys. Lett. B 26, 1250056 (2012).

${ }^{37}$ F. Huang, Z. Jiang, X. Lu, R. Ti, H. Wu, Y. Kan, and J. S. Zhu, Appl. Phys. Lett. 105, 022904 (2014).

${ }^{38}$ G. H. Haertling, J. Am. Ceram. Soc. 82, 797 (1999).

${ }^{39}$ W. Chen, X. Zhao, J. Sun, L. Zhang, and L. Zhong, J. Alloys Compd. 670, 48 (2016).

${ }^{40}$ P. P. Khirade, S. D. Birajdar, A. V. Raut, and K. M. Jadhav, Ceram. Int. 42, 12441 (2016).

${ }^{41}$ J. Tangsritrakul, M. Unruan, P. Ketsuwan, N. Triamnak, S. Rujirawat, T. Dechakupt, S. Anata, and R. Yimnirunet, Ferroelectrics 383, 166 (2009).

${ }^{42}$ Y. Shuai, S. Zhou, D. Bürger, H. Reuther, I. Skorupa, V. John, M. Helm, and H. Schmidt, J. Appl. Phys. 109, 084105 (2011).

${ }^{43}$ J. F. Scott and M. Dawber, Appl. Phys. Lett. 76, 3801 (2000).

${ }^{44}$ K. J. Choi, M. Biegalski, Y. L. Li, A. Sharan, J. Schubert, R. Uecker, P. Reiche, Y. B. Chen, X. Q. Pan, V. Gopalan, L. Q. Chen, D. G. Schlom, and C. B. Eom, Science 306, 1005 (2004).

${ }^{45}$ X. F. Wu, D. Vanderbilt, and D. R. Hamann, Phys. Rev. B 72, 035105 (2005).

${ }^{46}$ B. Xu, D. Wang, J. Íñiguez, and L. Bellaiche, Adv. Funct. Mater. 25, 552 (2015).

${ }^{47}$ S. Wada, S. Suzuki, T. Noma, T. Suzuki, M. Osada, M. Kakihana, S.-E. Park, L. E. Cross, and T. R. Shrout, Jpn. J. Appl. Phys., Part 1 38, 5505 (1999).

${ }^{48}$ P. W. Anderson, Phys. Rev. 124, 41 (1961).

${ }^{49}$ J. Hubbard, Proc. R. Soc. A 276, 238 (1963).

${ }^{50}$ E. Patino and A. Stashans, Ferroelectrics 256, 189 (2001).

${ }^{51}$ H. Ihrig, J. Phys. C: Solid State Phys. 11, 819 (1978).

${ }^{52}$ H. J. Hagemann and H. Ihrig, Phys. Rev. B 20, 3871 (1979).
${ }^{53}$ Y. B. Ma, A. Grünebohm, K. C. Meyer, K. Albe, and B. X. Xu, Phys. Rev. B 94, 094113 (2016).

${ }^{54}$ P. Murugaraj, T. R. N. Kutty, and M. S. Rao, J. Mater. Sci. 21, 3521 (1986).

${ }^{55}$ S. Y. Qiu, L. Wang, Y. Liu, Y. Q. Wu, and N. Chen, Trans. Nonferrous Met. Soc. China 20, 1911 (2010).

${ }^{56}$ D. P. Dutta, M. Roy, N. Maiti, and A. K. Tyagi, Phys. Chem. Chem. Phys. 18, 9758 (2016).

${ }^{57}$ The main component of $0.5 \mathrm{Ba}\left(\mathrm{Zr}_{0.2} \mathrm{Ti}_{0.8}\right) \mathrm{O}_{3}-0.5\left(\mathrm{Ba}_{0.7} \mathrm{Ca}_{0.3}\right) \mathrm{TiO}_{3}$ is $\mathrm{BaTiO}_{3}$, which explains why we have compatible results.

${ }^{58}$ J. M. Vielma and G. Schneider, J. Appl. Phys. 114, 174108 (2013).

${ }^{59}$ T. Nishimatsu, M. Iwamoto, Y. Kawazoe, and U. V. Waghmare, Phys. Rev. B 82, 134106 (2010).

${ }^{60}$ Additional tuning will be necessary to shift this potential morphortropic boundary to room temperature for practical applications.

${ }^{61}$ Z. Yu, R. Guo, and A. S. Bhalla, J. Appl. Phys. 88, 410 (2000).

${ }^{62}$ S. J. Kuang, X. G. Tang, L. Y. Li, Y. P. Jiang, and Q. X. Liu, Scr. Mater. 61, 68 (2009).

${ }^{63}$ W. Hu, Y. Liu, R. L. Withers, T. J. Frankcombe, L. Norén, A. Snashall, M. Kitchin, P. Smith, B. Gong, H. Chen, J. Schiemer, F. Brink, and J. W. Leung, Nat. Mater. 12, 821 (2013).

${ }^{64}$ D. M. Smyth, The Defect Chemistry of Metal Oxides (Oxford University Press, 2000).

${ }^{65}$ S. O. Kasap, Principles of Electronic Materials and Devices, 3rd ed. (McGraw-Hill, New York, 2006).

${ }^{66}$ The parameter, $\gamma$, of the effective Hamiltonian is negative. ${ }^{59}$ It indicates that the energy terms $u_{x}^{2} u_{y}^{2}, u_{y}^{2} u_{z}^{2}$, and $u_{x}^{2} u_{z}^{2}$ are energetically favored, which implies that the $\langle 111\rangle$ and $\langle 110\rangle$ type of dipoles are more likely than the $\langle 100\rangle$ type when only self-energy is considered. 